\title{
A MEAN ERGODIC THEOREM FOR ACTIONS OF AMENABLE QUANTUM GROUPS
}

\author{
ROCCO DUVENHAGE
}

\author{
(Received 11 September 2007)
}

\begin{abstract}
We prove a weak form of the mean ergodic theorem for actions of amenable locally compact quantum groups in the von Neumann algebra setting.

2000 Mathematics subject classification: primary 46L55; secondary 37A30.

Keywords and phrases: mean ergodic theorem, amenable quantum groups, von Neumann algebras.
\end{abstract}

\section{Introduction}

The following mean ergodic theorem is well known. Let $G$ be a locally compact group with right Haar measure $\mu$, and assume that it contains a Følner net $\left(\Lambda_{\lambda}\right)$, that is, a net of Borel sets in $G$ such that $0<\mu\left(\Lambda_{\lambda}\right)<\infty$ and $\lim _{\lambda} \mu\left(\Lambda_{\lambda} \Delta\left(\Lambda_{\lambda} g\right)\right) / \mu\left(\Lambda_{\lambda}\right)=0$ for all $g \in G$. Furthermore, let $U_{g}$ be a contraction on a Hilbert space $H$ such that $U_{g} U_{h}=U_{g h}$ for all $g, h \in G$, and $G \ni g \mapsto\left\langle U_{g} x, y\right\rangle$ is Borel measurable for all $x, y \in H$. Take $P$ to be the projection of $H$ onto $V:=\left\{x \in \mathfrak{H} \mid U_{g} x=x\right.$ for all $g \in G\}$. Then

$$
\lim _{\lambda} \frac{1}{\mu\left(\Lambda_{\lambda}\right)} \int_{\Lambda_{\lambda}} U_{g} x d \mu(g)=P x
$$

for all $x \in H$. A standard proof for the case $G=\mathbb{Z}$ can be found for example in [5] and [11], but it can be extended to the more general case without much effort (see the review in [2, Section 2]). A more general theorem in Banach spaces is presented in [10, Theorem (5.7)].

In this paper we prove a version of this theorem for the action of an amenable locally compact quantum group on a von Neumann algebra. We use the von Neumann algebra setting for quantum groups, as developed by Kustermans and Vaes [9] building on earlier work on Kac algebras (see, for example, [4]).

In this setting a locally compact quantum group is defined to be a von Neumann algebra $M$ with a unital normal $*$-homomorphism $\Delta: M \rightarrow M \otimes M$ (where $M \otimes$ $N$ denotes the von Neumann algebraic tensor product of two von Neumann

(c) 2008 Australian Mathematical Society 0004-9727/08 \$A2.00+0.00 
algebras), such that $\left(\Delta \otimes \iota_{M}\right) \circ \Delta=\left(\iota_{M} \otimes \Delta\right) \circ \Delta$ (where $\iota_{M}$ denotes the identity map on $M)$, and on which there exist normal semi-finite faithful weights $\varphi$ and $\psi$ such that $\varphi\left(\left(\theta \otimes \iota_{M}\right) \circ \Delta(a)\right)=\varphi(a) \theta(1)$ for all $a \in \mathcal{M}_{\varphi}^{+}$and $\psi\left(\left(\iota_{M} \otimes \theta\right) \circ \Delta(a)\right)=$ $\psi(a) \theta(1)$ for all $a \in \mathcal{M}_{\psi}^{+}$, for all $\theta \in M_{*}^{+}$, where $M_{*}^{+}$is set of the positive normal linear functionals on $M$, and $\mathcal{M}_{\varphi}^{+}=\left\{a \in M^{+} \mid \varphi(a)<\infty\right\}$. This quantum group is denoted as $(M, \Delta)$. We refer the reader to Kustermans and Vaes [6-8] for background and motivation for this definition. If, furthermore, there exists a net $\left(\varphi_{\lambda}\right)$ of normal states on $M$ such that $\left\|\theta * \varphi_{\lambda}-\varphi_{\lambda}\right\|$ converges to 0 for all $\theta \in M_{*}$ with $\theta(1)=1$, then we call $(M, \Delta)$ amenable; see, for example, [3]. Here $\mu * v:=(\mu \otimes v) \circ \Delta$ for any $\mu, \nu \in M_{*}^{+}$.

An action of $(M, \Delta)$ on another von Neumann algebra $A$ is defined to be a normal injective unital $*$-homomorphism $\alpha: A \rightarrow M \otimes A$ such that $\left(\iota_{M} \otimes \alpha\right) \circ$ $\alpha=\left(\Delta \otimes \iota_{A}\right) \circ \alpha$; see [14].

Given such an action, we shall assume the presence of a normal state $\omega$ on $A$ which is invariant under the action, by which we mean that $(\theta \otimes \omega) \circ \alpha=\omega$ for all normal states $\theta$ on $M$. In Section 2 we show how to set up the analogue of the integral in (1.1) for a quantum group action, and in Section 3 we state and prove a mean ergodic theorem for such actions, though only in a weak form analogous to

$$
\lim _{\lambda}\left\langle x, \frac{1}{\mu\left(\Lambda_{\lambda}\right)} \int_{\Lambda_{\lambda}} U_{g} y d \mu(g)\right\rangle=\langle x, P y\rangle
$$

for all $x, y \in H$. Our approach is to set the problem up in a suitable Hilbert space framework, closely related to that of (1.1), and then to follow the basic structure of the proof of (1.1).

We shall not need the full force of the theory of locally compact quantum groups as developed in $[8,9]$, and therefore it will be convenient to formulate our results in an abstract setting incorporating only the concepts from locally compact quantum groups that we need, modelled on the definitions discussed above. We shall focus on this abstract setting, rather than on concrete examples.

\section{A suitable integration theory}

In this section we develop the tools and notation that we need in order to formulate and prove the mean ergodic theorem in the next section. Throughout this section and the next we shall use the following notation: $R$ will be an arbitrary von Neumann algebra, its unit denoted by $1_{R}$, and its normal states by $\left(R_{*}^{+}\right)_{1}$. By $\omega$ we shall mean an arbitrary normal state on a von Neumann algebra $A$. We shall denote the GNS construction of $(A, \omega)$ by $(H, \gamma)$, by which we mean that $H$ is a Hilbert space and $\gamma: A \rightarrow H$ a linear mapping such that $\langle\gamma(a), \gamma(b)\rangle=\omega\left(a^{*} b\right)$ and with $\gamma(A)$ dense in $H$.

We remind the reader that we shall use the notation $R \otimes A$ to indicate the von Neumann algebraic tensor product, often written as $R \bar{\otimes} A$ in the literature. The algebraic tensor product will be written as $R \odot A$. We shall constantly use tensor 
products of mappings on von Neumann algebras, and a useful reference for this topic is [13]. For example, if $\theta$ is a normal state on $R$ while $\iota_{A}$ is the identity map $A \rightarrow A$, then we can define $\theta \otimes \iota_{A}: R \otimes A \rightarrow A$ as the tensor product of conditional expectations, in which case $\theta \otimes \iota_{A}$ itself is a conditional expectation, which is also normal, that is, $\sigma$-weakly continuous; see [13, Section 9].

We shall view $R$ as a noncommutative measurable space, and, roughly speaking, we shall be integrating $A$-valued 'functions' over $R$.

Note that the integral in (1.1) is an integral of a bounded function $f: G \rightarrow H$ which can be defined via the Riesz representation theorem by

$$
\left\langle\int_{\Lambda} f d \mu, x\right\rangle=\int_{\Lambda}\langle f(g), x\rangle d \mu(g) .
$$

We now mimic this construction for $A$-valued 'functions' on $R$, in other words for elements of $R \otimes A$.

PROPOSITION 2.1. Let $\mu$ be a normal positive linear functional on $R$. Then there is a unique function

$$
\widetilde{\mu}: R \otimes A \rightarrow H
$$

such that

$$
\langle\gamma(d), \tilde{\mu}(T)\rangle=\mu \otimes \omega\left(\left[1_{R} \otimes d\right]^{*} T\right)
$$

for all $T \in R \otimes A$ and $d \in A$. Furthermore, $\tilde{\mu}$ is linear, $\|\tilde{\mu}\| \leq\|\mu\|$ and

$$
\langle\gamma(d), \tilde{\mu}(T)\rangle=\omega\left(d^{*}\left(\mu \otimes \iota_{A}\right)(T)\right)
$$

for all $T \in R \otimes A$ and $d \in A$.

PROOF. For any $T \in R \otimes A$, define the linear functional

$$
f_{T}: \gamma(A) \rightarrow \mathbb{C}: \gamma(d) \mapsto \overline{\mu \otimes \omega\left(\left[1_{R} \otimes d\right]^{*} T\right)}
$$

which is indeed well defined, since if $\gamma(d)=0$ then $\mu \otimes \omega\left(\left[1_{R} \otimes d\right]^{*} T\right)=0$ as follows. First consider any

$$
T=\sum_{j=1}^{n} r_{j} \otimes a_{j} \in R \odot A
$$

then

$$
\left|\mu \otimes \omega\left(\left[1_{R} \otimes d\right]^{*} T\right)\right| \leq \sum_{j=1}^{n}\left|\mu\left(r_{j}\right)\right|\left|\omega\left(d^{*} a_{j}\right)\right|
$$

but

$$
\left|\omega\left(d^{*} a_{j}\right)\right| \leq \sqrt{\omega\left(d^{*} d\right)} \sqrt{\omega\left(a_{j}^{*} a_{j}\right)}=0,
$$

since $\omega\left(d^{*} d\right)=\|\gamma(d)\|^{2}$, therefore $\mu \otimes \omega\left(\left[1_{R} \otimes d\right]^{*} T\right)=0$. For a general $T \in R \otimes A$ there is a net $T_{\lambda} \in R \odot A$ converging $\sigma$-weakly to $T$, according to 
von Neumann's density theorem (see, for example, [1, Section 2.4.2]). Hence, $\left[1_{R} \otimes d\right]^{*} T_{\lambda}$ converges $\sigma$-weakly to $\left[1_{R} \otimes d\right]^{*} T$, but $\mu \otimes \omega$ is $\sigma$-weakly continuous (that is, normal), so $\mu \otimes \omega\left(\left[1_{R} \otimes d\right]^{*} T\right)=0$.

Clearly $f_{T}$ is linear, and $\left\|f_{T}\right\| \leq\|\mu\|\|T\|$ since

$$
\begin{aligned}
\left|f_{T}(\gamma(d))\right| & \leq \sqrt{\mu \otimes \omega\left(\left[1_{R} \otimes d\right]^{*}\left[1_{R} \otimes d\right]\right)} \sqrt{\mu \otimes \omega\left(T^{*} T\right)} \\
& \leq \sqrt{\mu\left(1_{R}\right)} \sqrt{\omega\left(d^{*} d\right)} \sqrt{\|\mu \otimes \omega\|\left\|T^{*} T\right\|} \\
& =\sqrt{\|\mu\|\|\gamma(d)\| \sqrt{\|\mu\|}\|T\| .}
\end{aligned}
$$

Therefore $f_{T}$ can be linearly extended uniquely to $H$ without changing its norm. By the Riesz representation theorem and since $\gamma(A)$ is dense in $H$, there is a unique element $\tilde{\mu}(T)$ in $H$ such that $f_{T}(\gamma(d))=\langle\tilde{\mu}(T), \gamma(d)\rangle$ for all $d \in A$. Furthermore, $\|\tilde{\mu}(T)\|=\left\|f_{T}\right\|$. Hence, we obtain a unique function $\tilde{\mu}: R \otimes A \rightarrow H$ such that (2.1) holds. Clearly $\tilde{\mu}$ is linear and $\|\tilde{\mu}(T)\| \leq\|\mu\|\|T\|$.

Lastly, for $r \in R$ and $a \in A$,

$$
\mu \otimes \omega\left(\left[1_{R} \otimes d\right]^{*}(r \otimes a)\right)=\omega\left(d^{*}\left(\mu \otimes \iota_{A}\right)(r \otimes a)\right),
$$

hence

$$
\mu \otimes \omega\left(\left[1_{R} \otimes d\right]^{*} T\right)=\omega\left(d^{*}\left(\mu \otimes \iota_{A}\right) T\right)
$$

for all $T \in R \odot A$ by linearity. But again by $\sigma$-denseness, and by $\sigma$-weak continuity, this extends to all $T \in R \otimes A$.

We now take this a step further by finding an analogue of the linear operator $H \rightarrow H: x \mapsto \int_{\Lambda} U_{g} x d \mu(g)$ that appears in (1.1).

PROPOSITION 2.2. Consider the situation in Proposition 2.1 and furthermore assume that we have a $*$-homomorphism $\alpha: A \rightarrow R \otimes A$ which leaves $\omega$ invariant in the sense that

$$
(\mu \otimes \omega) \circ \alpha=\mu\left(1_{R}\right) \omega
$$

for the given $\mu$. Then there exists a unique linear operator $\tilde{\mu}^{\alpha}: H \rightarrow H$ such that

$$
\tilde{\mu}^{\alpha}(\gamma(a))=\tilde{\mu} \circ \alpha(a)
$$

for all $a \in A$. Furthermore, $\left\|\tilde{\mu}^{\alpha}\right\| \leq\|\mu\|$, and if $\alpha$ is unital, then $\left\|\tilde{\mu}^{\alpha}\right\|=\|\mu\|$.

PRoOF. The operator $\tilde{\mu}^{\alpha}$ is well defined on $\gamma(A)$ since $\tilde{\mu} \circ \alpha(a)=0$ when $\gamma(a)=0$, as we now show. For any $d \in A$, from Proposition 2.1,

$$
\begin{aligned}
|\langle\gamma(d), \tilde{\mu} \circ \alpha(a)\rangle|^{2} & =\left|\mu \otimes \omega\left(\left[1_{R} \otimes d\right]^{2} \alpha(a)\right)\right|^{2} \\
& \leq \mu \otimes \omega\left(\left[1_{R} \otimes d\right]^{*}\left[1_{R} \otimes d\right]\right) \mu \otimes \omega\left(\alpha\left(a^{*} a\right)\right) \\
& =0
\end{aligned}
$$

by (2.2) and since $\omega\left(a^{*} a\right)=\|\gamma(a)\|^{2}=0$. But $\gamma(A)$ is dense in $H$, so $\tilde{\mu} \circ \alpha(a)=0$. Clearly $\tilde{\mu}^{\alpha}$ is linear and, as in the above calculation, for any $a, d \in A$, 


$$
\left|\left\langle\gamma(d), \tilde{\mu}^{\alpha}(\gamma(a))\right\rangle\right| \leq \mu\left(1_{R}\right)\|\gamma(d)\|\|\gamma(a)\|
$$

so $\left\|\widetilde{\mu}^{\alpha}\right\| \leq \mu\left(1_{R}\right)=\|\mu\|$. Hence, $\widetilde{\mu}^{\alpha}$ has a unique bounded linear extension to $H$, with the same norm. If $\alpha$ is unital, then by (2.1),

$$
\begin{aligned}
\left\langle\gamma(d), \tilde{\mu}^{\alpha}\left(\gamma\left(1_{A}\right)\right)\right\rangle & =\left\langle\gamma(d), \tilde{\mu}\left(1_{R} \otimes 1_{A}\right)\right\rangle \\
& =\mu\left(1_{R}\right) \omega\left(d^{*} 1_{A}\right) \\
& =\left\langle\gamma(d), \mu\left(1_{R}\right) \gamma\left(1_{A}\right)\right\rangle
\end{aligned}
$$

so $\widetilde{\mu}^{\alpha}\left(\gamma\left(1_{A}\right)\right)=\mu\left(1_{R}\right) \gamma\left(1_{A}\right)$ from which $\left\|\widetilde{\mu}^{\alpha}\right\|=\|\mu\|$ follows.

Lastly we shall need the following important property in the proof of the mean ergodic theorem. Note that by a normal $*$-homomorphism from one von Neumann algebra to another, we mean a $*$-homomorphism that is $\sigma$-weakly continuous.

Proposition 2.3. Consider the situation in Propositions 2.1 and 2.2. Furthermore, let $v$ be another normal positive linear functional on $R$ satisfying $(v \otimes \omega) \circ$ $\alpha=v\left(1_{R}\right) \omega$. Also assume that $\alpha$ is normal, and that $\Delta: R \rightarrow R \otimes R$ is a normal *-homomorphism such that

$$
\left(\iota_{R} \otimes \alpha\right) \circ \alpha=\left(\Delta \otimes \iota_{A}\right) \circ \alpha .
$$

Write

$$
\mu * v:=(\mu \otimes v) \circ \Delta
$$

Then it follows that

$$
\widetilde{\mu * \nu}^{\alpha}=\widetilde{v}^{\alpha} \widetilde{\mu}^{\alpha} .
$$

Proof. For any $r \in R$ and $a, d \in A$,

$$
\begin{aligned}
\omega\left(d^{*}\left[\mu \otimes\left(\nu \otimes \iota_{A}\right)\right] \circ\left(\iota_{R} \otimes \alpha\right)(r \otimes a)\right) & =\omega\left(d^{*} \mu(r)\left(\nu \otimes \iota_{A}\right) \circ \alpha(a)\right) \\
& =\langle\gamma(d), \widetilde{v} \circ \alpha(\mu(r) a)\rangle \\
& =\left\langle\gamma(d), \widetilde{v}^{\alpha}(\widetilde{\mu}(r \otimes a))\right\rangle
\end{aligned}
$$

by Propositions 2.1 and 2.2, hence by linearity

$$
\omega\left(d^{*}\left[\mu \otimes\left(\nu \otimes \iota_{A}\right)\right] \circ\left(\iota_{R} \otimes \alpha\right)(T)\right)=\left\langle\left(\widetilde{v}^{\alpha}\right)^{*} \gamma(d), \tilde{\mu}(T)\right\rangle
$$

for all $T \in R \odot A$. The left-hand side of (2.3) is a $\sigma$-weakly continuous linear functional of $T \in R \otimes A$, since $\iota_{R} \otimes \alpha$ is the tensor product of two $\sigma$-weakly continuous $*$-homomorphisms, and $(\mu /\|\mu\|) \otimes\left((\nu /\|v\|) \otimes \iota_{A}\right)$ that of two $\sigma$-weakly continuous conditional expectations (the case $\mu=0$ or $\nu=0$ being trivial). The righthand side of (2.3) is also a $\sigma$-weakly continuous linear functional of $T \in R \otimes A$. To see this, consider any net $T_{\lambda} \in R \otimes A$ converging $\sigma$-weakly to $T$. For any $c \in A$,

$$
\left\langle\gamma(c), \tilde{\mu}\left(T_{\lambda}\right)\right\rangle=\mu \otimes \omega\left(\left[1_{R} \otimes c\right]^{*} T_{\lambda}\right) \rightarrow \mu \otimes \omega\left(\left[1_{R} \otimes c\right]^{*} T\right)=\langle\gamma(c), \tilde{\mu}(T)\rangle
$$


in the $\lambda$ limit, since $\mu \otimes \omega$ is $\sigma$-weakly continuous. However, the $\sigma$-weak topology is a weak* topology, hence by the resonance theorem (uniform boundedness) the net ( $\left.T_{\lambda}\right)$ is bounded in the norm of $R \otimes A$. Since $\gamma(A)$ is dense in $H$, it therefore follows that $\left\langle x, \tilde{\mu}\left(T_{\lambda}\right)\right\rangle \rightarrow\langle x, \tilde{\mu}(T)\rangle$ for all $x \in H$, so indeed the right-hand side of (2.3) is $\sigma$-weakly continuous in $T$. But $R \odot A$ is $\sigma$-weakly dense in $R \otimes A$, therefore (2.3) holds for all $T \in R \otimes A$, in particular for $T=\alpha(a)$, so

$$
\begin{aligned}
\left\langle\gamma(d), \widetilde{v}^{\alpha} \tilde{\mu}^{\alpha}(\gamma(a))\right\rangle & =\omega\left(d^{*}\left[\mu \otimes\left(\nu \otimes \iota_{A}\right)\right] \circ\left(\iota_{R} \otimes \alpha\right) \circ \alpha(a)\right) \\
& =\omega\left(d^{*}\left[(\mu \otimes \nu) \otimes \iota_{A}\right] \circ\left(\Delta \otimes \iota_{A}\right) \circ \alpha(a)\right) \\
& =\omega\left(d^{*}\left\{[(\mu \otimes \nu) \circ \Delta] \otimes \iota_{A}\right\} \circ \alpha(a)\right) \\
& =\left\langle\gamma(d), \widetilde{\mu * \nu}^{\alpha}(\gamma(a))\right\rangle
\end{aligned}
$$

for any $a \in A$, by Propositions 2.1 and 2.2, and since $\Delta$ is normal (which ensures that $\left[(\mu \otimes v) \otimes \iota_{A}\right] \circ\left(\Delta \otimes \iota_{A}\right)=[(\mu \otimes v) \circ \Delta] \otimes \iota_{A}$ on $\left.R \otimes A\right)$. Since $\gamma(A)$ is dense in $H$, we obtain $\widetilde{v}^{\alpha} \widetilde{\mu}^{\alpha}=\widetilde{\mu * v}^{\alpha}$.

\section{The mean ergodic theorem}

Continuing with the notation of Section 2, we can now formulate and prove a mean ergodic theorem.

THEOREM 3.1. Consider two normal *-homomorphisms $\Delta: R \rightarrow R \otimes R$ and $\alpha: A \rightarrow R \otimes A$ such that $\left(\iota_{R} \otimes \alpha\right) \circ \alpha=\left(\Delta \otimes \iota_{A}\right) \circ \alpha$ and $(\theta \otimes \omega) \circ \alpha=\omega$ for all $\theta \in\left(R_{*}^{+}\right)_{1}$. Assume the existence of a net $\left(\varphi_{\lambda}\right)$ in $\left(R_{*}^{+}\right)_{1}$ such that $\left\|\theta * \varphi_{\lambda}-\varphi_{\lambda}\right\| \rightarrow 0$ for all $\theta \in\left(R_{*}^{+}\right)_{1}$. Let $P$ be the projection of $H$ on

$$
V:=\left\{x \in H \mid \tilde{\theta}^{\alpha} x=x \text { for all } \theta \in\left(R_{*}^{+}\right)_{1}\right\} .
$$

Then

$$
\lim _{\lambda}\left\langle x, \widetilde{\varphi}_{\lambda}^{\alpha} y\right\rangle=\langle x, P y\rangle
$$

for all $x, y \in H$.

PROOF. Set

$$
N=\overline{\operatorname{span}\left\{x-\widetilde{\theta}^{\alpha} x: x \in H, \theta \in\left(R_{*}^{+}\right)_{1}\right\}}
$$

and note that $\left\|\tilde{\theta}^{\alpha}\right\| \leq\|\theta\|=1$ (that is, $\tilde{\theta}^{\alpha}$ is a contraction). Then by a standard argument $N=V^{\perp}$ (see, for example, [5, Section 1.1]). Keep in mind that $(R \otimes$ $A)_{*}=R_{*} \otimes_{*} A_{*}$ where by $\otimes_{*}$ we mean the tensor product of Banach spaces with the completion taken in the dual norm of the spatial $\mathrm{C}^{*}$-norm on $R \odot A$ (see for example [12, Section 1.22]); this will be useful in the following calculation. Note that this dual norm is a cross norm. For any $a, d \in A$ and $\theta \in\left(R_{*}^{+}\right)_{1}$ it follows from Proposition 2.3 that 


$$
\begin{aligned}
& \left|\left\langle\gamma(d), \widetilde{\varphi}_{\lambda}^{\alpha}\left(\gamma(a)-\tilde{\theta}^{\alpha} \gamma(a)\right)\right\rangle\right| \\
& \quad=\left|\left\langle\gamma(d), \widetilde{\varphi}_{\lambda}^{\alpha} \gamma(a)-{\widetilde{\theta * \varphi_{\lambda}}}^{\alpha} \gamma(a)\right\rangle\right| \\
& \quad=\left|\varphi_{\lambda} \otimes \omega\left(\left[1_{R} \otimes d\right]^{*} \alpha(a)\right)-\left(\theta * \varphi_{\lambda}\right) \otimes \omega\left(\left[1_{R} \otimes d\right]^{*} \alpha(a)\right)\right| \\
& \quad=\left|\left(\varphi_{\lambda}-\theta * \varphi_{\lambda}\right) \otimes \omega\left(\left[1_{R} \otimes d\right]^{*} \alpha(a)\right)\right| \\
& \quad \leq\left\|\varphi_{\lambda}-\theta * \varphi_{\lambda}\right\|\|\omega\|\left\|\left[1_{R} \otimes d\right]^{*} \alpha(a)\right\| \\
& \quad \rightarrow 0 .
\end{aligned}
$$

Furthermore, $\left\|\widetilde{\varphi}_{\lambda}^{\alpha}-\widetilde{\varphi}_{\lambda}^{\alpha} \widetilde{\theta}^{\alpha}\right\| \leq 2$ by Proposition 2.2 , so $\left(\widetilde{\varphi}_{\lambda}^{\alpha}-\widetilde{\varphi}_{\lambda}^{\alpha} \widetilde{\theta}^{\alpha}\right)$ is a bounded net, while $\gamma(A)$ is dense in $H$, hence

$$
\left\langle x, \widetilde{\varphi}_{\lambda}^{\alpha}\left(y-\widetilde{\theta}^{\alpha} y\right)\right\rangle \rightarrow 0
$$

for all $x, y \in H$ and $\theta \in\left(R_{*}^{+}\right)_{1}$. Since $\left\|\widetilde{\varphi}_{\lambda}^{\alpha}\right\| \leq 1$, we conclude from the definition of $N$ that

$$
\left\langle x, \widetilde{\varphi}_{\lambda}^{\alpha} y\right\rangle \rightarrow 0
$$

for all $x \in H$ and all $y \in N$. So for any $x, y \in H$ we obtain

$$
\begin{aligned}
\left\langle x, \widetilde{\varphi}_{\lambda}^{\alpha} y\right\rangle & =\left\langle x, \widetilde{\varphi}_{\lambda}^{\alpha} P y\right\rangle+\left\langle x, \widetilde{\varphi}_{\lambda}^{\alpha}(1-P) y\right\rangle \\
& =\langle x, P y\rangle+\left\langle x, \widetilde{\varphi}_{\lambda}^{\alpha}(1-P) y\right\rangle \\
& \rightarrow\langle x, P y\rangle
\end{aligned}
$$

by the definition of $P$ and since $(1-P) y \in V^{\perp}=N$.

In particular, this result holds in the situation presented in Section 1, where $R=M$ is an amenable locally compact quantum group. This is our main and final result, and we now conclude with a few brief remarks to give some indication of the relation with classical ergodic theory and how the theorem might be used.

Note that if $\alpha$ is unital in Theorem 3.1, then $P \Omega=\Omega$, where $\Omega:=\gamma\left(1_{A}\right)$ is the (nonzero) cyclic vector of the GNS construction of $(A, \omega)$, since

$$
\left\langle\gamma(d), \tilde{\theta}^{\alpha} \Omega\right\rangle=\left\langle\gamma(d), \tilde{\theta}\left(1_{R} \otimes 1_{A}\right)\right\rangle=\langle\gamma(d), \Omega\rangle
$$

for all $\theta \in\left(R_{*}^{+}\right)_{1}$. This is essentially the same situation as in classical ergodic theory. Extending the classical case, it seems reasonable to say that the dynamical system $(A, \omega, \alpha)$ is ergodic when $\operatorname{dim} P H=1$, that is, $P H=\mathbb{C} \Omega$. Using Theorem 3.1, this is easily seen to be equivalent to

$$
\lim _{\lambda} \varphi_{\lambda} \otimes \omega\left(\left[1_{R} \otimes a\right] \alpha(b)\right)=\omega(a) \omega(b),
$$

again paralleling the situation in classical ergodic theory.

One simple application of the mean ergodic theorem in classical ergodic theory is Khintchine's recurrence theorem; see, for example, [11, Section 2.3]. If we only had the weak form (1.2) of the mean ergodic theorem, we would correspondingly get a 
weaker recurrence result, which we now discuss. For the simple case of an action of $\mathbb{Z}$ on a measure space $(X, \Sigma, \mu)$ given by a measure preserving transformation $T: X \rightarrow X$ this recurrence result can be expressed as follows: for any $S \in \Sigma, \varepsilon>0$ and $j \in \mathbb{Z}$ there is a $\lambda_{0} \in \mathbb{N}$ such that

$$
\frac{1}{\lambda+1} \sum_{n=j}^{j+\lambda} \mu\left(S \cap T^{-n} S\right)>\mu(S)^{2}-\varepsilon
$$

for all $\lambda \geq \lambda_{0}$. To clarify the interpretation of this as recurrence, note that it implies that $\mu\left(S \cap T^{-n} S\right)>\mu(S)^{2}-\varepsilon$ for some $n \in\left\{j, j+1, \ldots, j+\lambda_{0}\right\}$. The strong form of the mean ergodic theorem results in this recurrence taking place on a relatively dense (or syndetic) set. The latter result was generalized in [2] to a state-preserving action of a group on a noncommutative $*$-algebra. We now consider the action of a quantum group. For a dynamical system $(A, \omega, \alpha)$ as above, and the situation in Theorem 3.1, for any $a \in A, \varepsilon>0$ and $\theta \in\left(R_{*}^{+}\right)_{1}$ there is a $\lambda_{0}$ such that

$$
\begin{aligned}
& \left|\left\langle\gamma(a){\widetilde{\varphi_{\lambda} * \theta}}^{\alpha} \gamma(a)\right\rangle-\langle\gamma(a), P \gamma(a)\rangle\right| \\
& \quad=\left|\left\langle\left(\widetilde{\theta}^{\alpha}\right)^{*} \gamma(a), \widetilde{\varphi}_{\lambda}^{\alpha} \gamma(a)\right\rangle-\left\langle\left(\widetilde{\theta}^{\alpha}\right)^{*} \gamma(a), P \gamma(a)\right\rangle\right| \\
& \quad<\varepsilon
\end{aligned}
$$

for all $\lambda \geq \lambda_{0}$ according to Theorem 3.1. Hence

$$
\left|\left(\varphi_{\lambda} * \theta\right) \otimes \omega\left(\left[1_{R} \otimes a\right]^{*} \alpha(a)\right)\right|>|\omega(a)|^{2}-\varepsilon
$$

for all $\lambda \geq \lambda_{0}$. We could then interpret this as a form of recurrence, analogous to (3.1), with $a$ generalizing the characteristic function of $S, \omega$ generalizing integration with respect to $\mu$, and $\varphi_{\lambda} * \theta$ generalizing $1 /(\lambda+1) \sum_{n=j}^{j+\lambda}$ if we view $\varphi_{\lambda}$ as corresponding to the unshifted sum $1 /(\lambda+1) \sum_{n=0}^{\lambda}$.

\section{Acknowledgements}

I thank Johan Swart and Gusti van Zyl for useful conversations, as well as an anonymous referee for suggestions.

\section{References}

[1] O. Bratteli and D. W. Robinson, Operator Algebras and Quantum Statistical Mechanics, 2nd edn, Vol. 1 (Springer, New York, 1987).

[2] R. de Beer, R. Duvenhage and A. Ströh, 'Noncommutative recurrence over locally compact Hausdorff groups', J. Math. Anal. Appl. 322 (2006), 66-74.

[3] P. Desmedt, J. Quaegebeur and S. Vaes, 'Amenability and the bicrossed product construction', Illinois J. Math. 46 (2002), 1259-1277.

[4] M. Enock and J.-M. Schwartz, Kac Algebras and Duality of Locally Compact Groups (Springer, Berlin, 1992).

[5] U. Krengel, Ergodic Theorems (Walter de Gruyter and Co., Berlin, 1985). 
[6] J. Kustermans and S. Vaes, 'A simple definition for locally compact quantum groups', C. R. Acad. Sci. Paris Sér. I Math. 328 (1999), 871-876.

[7] , 'The operator algebra approach to quantum groups', Proc. Natl Acad. Sci. USA 97 (2000), 547-552.

[8] — - 'Locally compact quantum groups', Ann. Sci. École Norm. Sup. (4) 33 (2000), 837-934.

[9] - 'Locally compact quantum groups in the von Neumann algebraic setting', Math. Scand. 92 (2003), 68-92.

[10] A. L. T. Paterson, 'Amenability', in: Mathematical Surveys and Monographs, Vol. 29 (American Mathematical Society, Providence, RI, 1988).

[11] K. Petersen, Ergodic Theory (Cambridge University Press, Cambridge, 1983).

[12] S. Sakai, $C^{*}$-algebras and $W^{*}$-algebras (Springer, Berlin, 1998), reprint of the 1971 edition.

[13] Ş. Strătilă, Modular Theory in Operator Algebras (Editura Academiei Republicii Socialiste România, Abacus Press, Bucharest, Tunbridge Wells, 1981), translated from the Romanian by the author.

[14] S. Vaes, 'The unitary implementation of a locally compact quantum group action', J. Funct. Anal. 180 (2001), 426-480.

ROCCO DUVENHAGE, Department of Mathematics and Applied Mathematics, University of Pretoria, 0002 Pretoria, South Africa

e-mail: rocco.duvenhage@up.ac.za 